\title{
The use of optimised heating trousers and the role of the blood flow on the reduction in muscle temperature post warm up
}

\author{
Margherita Raccuglia*, Alex Lloyd, Davide Filingeri, Simon Hodder, George Havenith \\ From 15th International Conference on Environmental Ergonomics (ICEE XV) \\ Portsmouth, UK. 28 June - 3 July 2015
}

\begin{abstract}
Introduction
Activities that are highly dependent on power output can benefit from increases in muscle temperature $\left(T_{m}\right)$ in terms of work done and skeletal muscle power output. When athletes experience a significant delay between active warm up and performance, $\mathrm{T}_{\mathrm{m}}$ declines. Previous studies have demonstrated that using heated trousers during a period of inactivity can attenuate this decline, with a greater peak power output as result $[1,2]$. However, in these studies, the reduction in $\mathrm{T}_{\mathrm{m}}$ was not completely eliminated. Thus, in the current study we aimed to optimise the heating procedure, in order to eliminate the reduction in $\mathrm{T}_{\mathrm{m}}$ post-warm up. Furthermore, to understand the reason of this reduction, the effect of the blood flow in the cooling process of the leg was studied.
\end{abstract}

\section{Method}

Ten male cyclists participated in this experiment. The heating garment was applied during 30 minutes of passive recovery following 15 minutes of active warm up. The heating procedure was optimised by using water perfused trousers with an adjusted water temperature of $43{ }^{\circ} \mathrm{C}$. The effect of the blood flow was observed during the recovery period using full restriction of arterial and venous blood flow in one leg (OCCLUDED), while the other leg was used as control (CONTROL). $\mathrm{T}_{\mathrm{m}}$ of the vastus lateralis was measured at three different depths beyond the muscle fascia: $5 \mathrm{~mm}, 15 \mathrm{~mm}$ and $25 \mathrm{~mm}$.

\section{Results}

During the passive recovery, blood flow significantly reduced $(\mathrm{p}<0.05) \mathrm{T}_{\mathrm{m}}$ in CONTROL compared to

\footnotetext{
* Correspondence: M.Raccuglia2@lboro.ac.uk

Environmental Ergonomics Research Centre, Loughborough University, Loughborough UK
}

1. Faulkner SH, Ferguson RA, Gerrett N, Hupperets M, Hodder SG, Havenith G: Reducing muscle temperature drop after warm-up improves sprint cycling performance. Medicine and science in sport and exercise 2013, 45(2):359-65.

2. Faulkner SH, Ferguson RA, Hodder SG, Havenith G: External muscle heating during warm-up does not provide added performance benefit above external heating in recovery period alone. European journal of applied physiology 2013, 113(11):2713-21.

doi:10.1186/2046-7648-4-S1-A77

Cite this article as: Raccuglia et al:: The use of optimised heating trousers and the role of the blood flow on the reduction in muscle temperature post warm up. Extreme Physiology \& Medicine 2015 4(Suppl 1):A77. 